\title{
Implementing and assessing the value of nursing preceptorship
}

Odelius A, Traynor M, Mehigan S et al (2017) Implementing and assessing the value of nursing preceptorship. Nursing Management. 23, 9, 35-37. Date of submission: I5 June 2016; date of acceptance: 26 August 2016. doi: 10.7748/nm.2017.el547

\author{
Anki Odelius \\ Research fellow, University \\ of Surrey

\section{Michael Traynor Professor of nursing policy, and Education, Middlesex University, London} \\ Department of Adult, Child and \\ Midwifery, School of Health
}

\section{Sinead Mehigan} Head of department, Middlesex University, and programme manager, Capital Nurse, Health Education England

\section{Mercy Wasike Community and primary care nurse lead, Health Education England \\ Chris Caldwell \\ Director of nursing at the Tavistock and Portman NHS Foundation Trust, London, and programme director, Capital Nurse, Health Education England}

\section{Correspondence m.traynor@mdx.ac.uk}

\section{Conflict of interest None declared}

\section{Peer review}

This article has been subject to external double-blind peer review and checked for plagiarism using automated software

\begin{abstract}
Preceptorship was first promoted as part of the Project 2000 reforms, and evidence suggests it remains valuable to newly qualified nurses. Although there is a lack of research of its effect on patient care, what research there is highlights various positive aspects of preceptorship programmes for newly qualified nurses, preceptors, and organisations. This article summarises the results of a scoping review of the literature, published since 2009 , on preceptorship. It also describes a preceptorship initiative in London led by Health Education England.
\end{abstract}

\section{Keywords}

Capital Nurse, Health Education England, newly qualified nurses, nursing management, preceptorship

SINCE IT was first promoted as part of the Project 2000 reforms, the need for and the value of preceptorship for newly qualified nurses have been highlighted in policies, academic papers and the nursing press, including Health Education England's (HEE) Shape of Caring Review (HEE 2015). Many nurses report that peer support in the early months of registration is helpful and NHS organisations are expected to provide preceptorship programmes, sometimes with specific components, and to detailed standards such as those developed by the HEE (2015) (Box 1).

The challenge for any nursing initiative is a combination of: time, busyness and pressures, which are named continually as barriers to preceptorship. This article outlines current knowledge about preceptorship in nursing, and describes some promising approaches to implementation. It also includes some points intended to provoke discussion. The scoping review is part of the work of Capital Nurse, a coalition of commissioners, NHS managers and academics working on nursing workforce issues in London (Box 2).

The Capital Nurse programme has three overarching aims. The first is to ensure a collective approach to providing appropriately skilled nurses to meet the changing requirements of healthcare in London. The programme is also designed to help ensure there are structures and a workforce in place to deliver high-quality care across the capital and to work collectively to plan the transition to new methods of funding for pre-registration nursing education.
There are four activity streams in the programme: employment of graduating nursing students, career progression, minimising the use of agency staff, and the transition to new funding models for pre-registration nursing programmes. The career progression workstream is developing clearer career pathways for nurses, and the scoping review was commissioned as part of the work on earlystage career development. We are identifying and sharing best practice in relation to how newly qualified nurses are supported through their first months and encouraged to continue to work in London, using findings from the literature and information from employers.

The nursing workforce in London is not typical of the UK. Historically, the capital has a higher turnover than the rest of the country (Finlayson et al 2002), and the greatest percentage of unfilled nursing posts (Royal College of Nursing 2016). These issues create particular challenges for developing and delivering preceptorship programmes (Box 3).

\section{Scoping review}

The use of scoping reviews to synthesise research findings is increasing, although there is no common understanding of what the term 'scoping review' should mean (Colquhoun et al 2014). For this article, however, a scoping review is understood to mean a rapid criticalliterature review, intended to capture the breadth and depth of material related to preceptorship in nursing. The literature search was conducted in January and February 2016, and was limited to work related to 


\section{Online archive}

For related information, visit nursingmanagement. co.uk and search using the keywords preceptorship in nursing in the UK published from 2009 onwards.

A combined search term, 'newly AND qualified AND nurs* AND (UK OR United Kingdom) OR precept* AND nurs* AND (UK OR United Kingdom)', generated the most relevant results, using the British Nursing Index, Cumulative Index to Nursing and Allied Health Literature (CINAHL), MEDLINE and other databases. The search generated 523 results. All titles and abstracts were reviewed by one member of the research team, the members of which also reviewed articles when it was impossible to establish relevance from the abstract. After exclusions, the team agreed on 15 peer-reviewed articles, and one review report commissioned by the Department of Health (DH).

\section{Box 1. Health Education England preceptorship standards}

In 2015, Health Education England (HEE) adopted Health Education North Central and East London's pan-London preceptorship standards (2014) and the Health Education North West Learning and Development Agreement Schedule C Preceptorship Outcomes nationally. Both documents inform HEE education commissioners.

\section{Box 2. Capital Nurse}

Since July 2015, directors of nursing in London, staff from the London branch of NHS England and Health Education England (HEE), as well as other stakeholders, have been addressing the challenges of nursing workforce issues in the capital with a programme called Capital Nurse. The programme addresses some of the recommendations in the HEE's response to the Shape of Caring Review (HEE 2016), specifically those relating to the 'standards for postgraduate education' and 'excellence in nursing practice' themes. This scoping review of preceptorship is

\section{Box 3. The benefits and challenges of working in London}

A survey of newly qualified and final-year nursing students in London, carried out as part of the Capital Nurse programme, indicated that some of the benefits of working in London are the opportunity to work with a diverse workforce, in a diverse city, and of pioneering healthcare environments where practice and research are often cutting edge. London is also viewed as offering unique opportunities to develop nursing careers. Challenges include the high cost of living and travel, complicated recruitment processes, and long, sometimes inflexible, shift patterns. Some of the other issues identified include the need for improvements in induction and preceptorship programmes, and the need for some organisations to value staff more.

\section{Box 4. Reflection on good preceptorship}

The Health Education England, North Central and East London community and primary care nursing lead reflects on preceptorship:

'My preceptorship period was well structured and protected. We had clear and consistent support from senior clinical leaders, which meant we were always able to take that afternoon once a month to attend pre-planned sessions.

'Having a clear, well structured programme meant we knew what to expect from the organisation, ourselves, the teams we worked with and each other. The organisation had a named preceptorship lead who facilitated our sessions. This allowed us to create a safe space where we could grow by acquiring the skills we needed for transition and beyond.

'Two of the skills we worked on were communication, developing the ability to articulate our needs and suggest solutions, and coping with stress through the effective use of appropriate strategies while under pressure. The relationship we formed with the preceptorship lead was a sound foundation for clinical supervision, which continues to inform my practice nearly 15 years later.'

\section{Promising approaches}

To identify promising approaches, the team focused on three main themes that emerged from the literature review:

»Experiences of preceptees and preceptors.

» Taking a standardised or individual approach to preceptorship.

"Important components of successful transition.

Experiences of preceptees and preceptors Much has been written about how most newly qualified nurses appreciate periods of preceptorship and it is largely this that supports its value in the absence of more robust evidence, for example better recruitment and retention, or better patient care. There is also an emerging, but small, body of literature that focuses on preceptors' views. The perceived value of peer support for newly qualified nurses, alongside a supportive work culture, is a strong theme.

Many studies, for example MarksMaran et al (2013), support the general argument for preceptorship, in that most nurses who participate find it valuable for increasing their competence and confidence. Preceptees generally perceive that programmes have a positive effect on communication skills, personal development, relationships with colleagues, clinical skills, stress and anxiety. A minority do not find preceptorship useful because of poor relationships with preceptors, or lack of time. Some nurses, particularly in specialist clinical areas, find they do not need preceptorship because they receive the support they require from specialist colleagues.

\section{Approaches to preceptorship}

The review identified examples of preceptorship programme evaluations, representing broadly contrasting approaches. These evaluations or studies tended to be small and could be criticised for lack of rigour, for example in their approach to sampling. However, they describe some apparently promising practicebased solutions. For example, Jones et al (2014) suggest that centrally planned and coordinated programmes have practical advantages, because they allow for forward planning of time off to attend education sessions, and enable ward managers to identify when nurses have acquired certain skills. New nurses can find the peer support they experience through such programmes valuable.

In contrast, 'roll-on, roll-off preceptorship pathways' (Chapman 2013) offer flexible and individually adapted support to newly qualified nurses. This can avoid the problem of trying 
to release large numbers of staff at the same time, and is flexible in terms of acquiring the skills required for different practice areas. New nurses can be enrolled on a programme as soon as they begin working, and their educational needs and skills acquisition can be discussed with managers. Teaching sessions can be provided alongside preceptors, peer support, opportunities to reflect, and distance learning linked to the NHS Knowledge and Skills Framework (Department of Health 2004) and appraisal needs. Such approaches rely to a considerable extent on self-directed learning, assessment and extensive paperwork, which can be viewed negatively (Chapman 2013).

\section{Successful transition}

Effective support requires organisational commitment to developing frameworks that goes further than commitment on paper and could involve providing sufficient resources, appointing dedicated staff, and planning free time. However, lack of resources, such as staff shortages and demanding workloads, is the main challenge to delivering effective, organised support for new nurses (Robinson and Griffiths 2009).

Other important features, particularly for preceptees, are peer support and positive work cultures. Peer support is highly valued and regarded as the most important form of support, provides 'moral support', reduces stress in newly qualified nurses (Rush et al 2013) and boosts their confidence (Whitehead et al 2013, Jones et al 2014).

Although not strictly a feature of organised support for newly qualified nurses, positive and supportive work cultures are regarded as important for successful transition from student to practitioner (Rush et al 2013). To rely solely on one preceptor can be unrealistic and leave new nurses without support when they need it.
One example of an effective preceptorship process is given in Box 4.

\section{Points for discussion}

The following points are intended for discussion at local and strategic levels. Some are speculative and based on the authors' interpretations of findings from the scoping review and national policies:

»Preceptorship programmes are valuable, but organisations should not rely on them as solely in supporting newly registered nurses. They should also be clear about what preceptorship is and what it is not, what newly qualified nurses need, and what is possible in the organisation. Expectations may need to be modified to sustain local schemes.

"Organisations should invest in preceptors, and provide training, support, time and rewards.

» Regardless of how well preceptorship is implemented, the value of peer support and supportive work cultures cannot be over-emphasised. Continued high pressure, changes and uncertainty about staffing can corrupt the most positive of cultures.

"The repetition of the orthodoxy that newly qualified nurses are not confident and are 'unfinished' may become self-fulfilling. Nurses have qualified as autonomous registered practitioners and should not be regarded as practising in a 'twilight zone' of trust during preceptorship periods.

» Organisations should evaluate and improve preceptorship programmes regularly.

»Those planning preceptorship programmes should use workshop formats when possible, because they provide built-in peer support and foster independent thinking.

» Although research shows that newly qualified nurses appreciate the support provided by preceptorship, there is no published evidence to show that it improves patient care or the recruitment and retention of nurses.

\section{UPDATES}

The Capital Nurse programme can be followed on Twitter by using the hashtag \#CapitalNurse

\section{References}

Chapman L (2013) A 'roll-on, roll-off' preceptorship pathway for new registrants. Nursing Management. 20, 2, 24-26.

Colquhoun H, Levac D, O'Brien Ket al (2014) Scoping reviews: time for clarity in definition methods, and reporting. Journal of Clinical Epidemiology. 67, 1291-1294

Department of Health (2004) The NHS Knowledge and Skills Framework and the Development Review Process. Agenda for Change Project Team, Leeds.

Finlayson B, Dixon J Meadows S et al (2002) Mind the Gap: The Extent of the NHS Nursing Shortage. King's Fund, London.
Health Education England (2015) Raising the Bar. Shape of Caring: A Review of the Future Education and Training of Registered Nurses and Care Assistants. www.hee.nhs.uk/sites/default/ files/documents/2348-Shape-of-caring-reviewFINAL_0.pdf (Last accessed: 7 December 2016.)

Health Education England (2016) Raising the Bar: Shape of Caring: Health Education England's Response. www.hee.nhs.uk/sites/default/files/ documents/Raising\%20the\%20Bar\%20-\%20 Shape\%20of\%2OCaring\%20-\%20HEE\% $27 \mathrm{~s} \% 20$ response.pdf (Last accessed: 7 December 2016.)

Health Education North Central and East London
(2014) Preceptorship Standards. https://hee nhs.uk/sites/default/files/documents/NCELPreceptorship-Standards-2014.pdf (Last accessed: 7 December 2016.)

Jones A, Benbow J, Gidman R (2014) Provision of training and support for newly qualified nurses. Nursing Standard. 28, 19, 44-50.

Marks-Maran D, Ooms A, Tapping J et al (2013) A preceptorship programme for newly qualified nurses: a study of preceptees' perceptions. Nurse Education Today. 33, 1428-1434.

Royal College of Nursing (2016) Safe Staffing Report 2015. RCN, London.
Robinson S, Griffiths P (2009) Scoping Review: Preceptorship for Newly Qualified Nurses; Impacts, Facilitators, and Constraints. King's College, London.

Rush K, Adamack M, Gordon J et al (2013) Best practices of formal new graduate nurse transition programs: an integrative review. International Journal of Nursing Studies. 50, 345-356.

Whitehead B, Owen P, Holmes D et al (2013) Supporting newly qualified nurses in the UK: a systematic literature review. Nurse Education Today. 33, 370-377. 